\title{
Effective $Q$ factor formula for small spherical surface antennas
}

\author{
Keisuke Fujita ${ }^{\text {a) }}$ \\ Maebashi Institute of Technology, \\ 460-1 Kamisadori, Maebashi, Gunma 371-0816, Japan \\ a)fujita@maebashi-it.ac.jp
}

Abstract: This letter presents an effective Q factor formula for self-resonant spherical surface antennas. The self-resonant lossless $Q$ factor and radiation efficiency calculated using spherical wave expansion provide an approximated expression for the effective $\mathrm{Q}$ factor. The resultant effective $\mathrm{Q}$ factor is larger than that of the infinitesimal loop antenna and smaller than that of the infinitesimal dipole antenna. Comparison of the result with the Q factor of spherical helix antennas has shown good agreement. A simple estimation formula can help design a small spherical helix antenna.

Keywords: Q factor, small antenna, radiation efficiency

Classification: Antennas and Propagation

\section{References}

[1] A. D. Yaghjian and S. R. Best, "Impedance, bandwidth, and Q of antennas," IEEE Trans. Antennas Propag., vol. 53, no. 4, pp. 1298-1324, Apr. 2005. DOI:10.1109/TAP.2005.844443

[2] L. J. Chu, "Physical limitations of omni-directional antennas," J. Appl. Phys., vol. 19, no. 12, pp. 1163-1175, Dec. 1948. DOI:10.1063/1.1715038

[3] R. C. Hansen and R. E. Collin, "A new Chu formula for Q," IEEE Antennas Propag. Mag., vol. 51, no. 5, pp. 38-41, Oct. 2009. DOI:10.1109/MAP.2009. 5432037

[4] T. V. Hansen, O. S. Kim, and O. Breinbjerg, "Stored energy and quality factor of spherical wave functions - in relation to spherical antennas with material cores," IEEE Trans. Antennas Propag., vol. 60, no. 3, pp. 1281-1290, Mar. 2012. DOI:10.1109/TAP.2011.2180330

[5] M. Gustafsson and S. Nordebo, "Optimal antenna currents for Q, superdirectivity, and radiation patterns using convex optimization," IEEE Trans. Antennas Propag., vol. 61, no. 3, pp. 1109-1118, Mar. 2013. DOI:10.1109/ TAP.2012.2227656

[6] R. F. Harrington, "Effect of antenna size on gain, bandwidth, and efficiency," J. Res. Nat. Bur. Stand. Section D: Radio Propagation, vol. 64D, no. 1, pp. 1-12, Jan. 1960.

[7] A. D. Yaghjian and H. R. Stuart, "Lower bounds on the Q of electrically small dipole antennas," IEEE Trans. Antennas Propag., vol. 58, no. 10, pp. 31143121, Oct. 2010. DOI:10.1109/TAP.2010.2055790

[8] K. Fujita and H. Shirai, "Theoretical limit of the radiation efficiency for electrically small self-resonant spherical surface antennas," IEICE Trans. Electron., vol. E100-C, no. 1, pp. 20-26, Jan. 2017. DOI:10.1587/transele. 
E100.C.20

[9] C. Pfeiffer, "Fundamental efficiency limits for small metallic antennas," IEEE Trans. Antennas Propag., vol. 65, no. 4, pp. 1642-1650, Feb. 2017. DOI:10. 1109/TAP.2017.2670532

[10] H. L. Thal, "Radiation efficiency limits for elementary antenna shapes," IEEE Trans. Antennas Propag., vol. 66, no. 5, pp. 2179-2187, May 2018. DOI:10. 1109/TAP.2018.2809507

[11] K. Fujita, "Effective Q factor for spherical surface antennas," Proc. iWAT, Nanjing, China, pp. 1-3, Mar. 2018. DOI:10.1109/IWAT.2018.8379124

[12] J. D. Jackson, Classical Electrodynamics, 3rd ed., John Wiley \& Sons, New Jersey, 1999.

[13] C. A. Balanis, Antenna Theory: Analysis and Design, 3rd ed., John Wiley \& Sons, New Jersey, 2005.

[14] M. Capek and L. Jelinek, "Optimal composition of modal currents for minimal quality factor Q," IEEE Trans. Antennas Propag., vol. 64, no. 12, pp. 52305242, Dec. 2016. DOI:10.1109/TAP.2016.2617779

\section{Introduction}

The bandwidth and radiation efficiency of electrically small antennas are both strongly affected by a small antenna size. The channel capacity of the communication is limited by its bandwidth, thereby necessitating a wide operating bandwidth. As the bandwidth is proportional to the reciprocal of the Q factor [1], the bandwidth of electrically small antennas is evaluated using the Q factor.

The theoretical limit for the Q factor can be obtained both analytically and numerically. Chu [2] demonstrated that a small antenna fabricated using lossless materials cannot exceed the theoretical lower bound using the spherical wave expansion. This result is limited to the case wherein the stored electric and magnetic energy inside the circumscribing sphere is zero. The stored energy inside the sphere can be increased by expanding the electromagnetic field inside the sphere [3, 4]. The Q factor of arbitrarily shaped antennas have also been numerically calculated by discretizing the antenna surface and applying convex optimization [5].

The bandwidth of a small antenna should be evaluated by the effective Q factor rather than the lossless Q factor because the low radiation efficiency of a small antenna increases the effective bandwidth. The radiation efficiency and the effective Q factor of a gain-optimized spherical antenna have been derived by Harrington [6], but this publication does not mention the case for the maximum radiation efficiency. The effective Q factor of the non-resonant small antenna is analytically calculated using the radiation efficiency of the infinitesimal dipole and loop antenna [7]. The radiation efficiency of these antennas is underestimated for the small selfresonant antenna and is unsuitable for estimating the self-resonant effective Q factor.

A recent investigation has revealed that the upper bound of the radiation efficiency for the small self-resonant antenna can be obtained using the spherical wave expansion [8] and the equivalent circuit method $[9,10]$. The $\mathrm{Q}$ factor of the lossless antenna increases monotonically as the antenna size decreases. In contrast, the effective $\mathrm{Q}$ factor of small antennas with lossy material approaches zero owing 
to the low radiation efficiency [11]. This finding poses the question of how large the effective $\mathrm{Q}$ factor of the transition region should be to ensure a value between an extremely small antenna size and an intermediate one. To answer this question, we herein investigate the effective Q factor of the self-resonant spherical surface antenna for all small antenna regions using the lossless self-resonant Q factor and the radiation efficiency. These results may help the antenna designer by providing not only a design guideline for spherical helix antennas but also a rough estimate for general small antennas.

\section{Lossless $Q$ factor for self-resonant spherical surface antennas}

We assumed a spherical current sheet with a radius $R$ fabricated from a good conductor having conductivity $\sigma$ as the spherical surface antenna. The center of the spherical surface antenna coincides with the origin of the spherical coordinate system. The current distribution on the sphere and the radiated electromagnetic fields can be expressed using the vector spherical wave expansion [12] and classified into $\mathrm{TM}_{n m}$ or $\mathrm{TE}_{n m}$ modes, where $n$ and $m$ denote the indices in the radial and azimuthal directions, respectively. As a small antenna was considered, it was assumed that the lowest-mode $(n=1)$ current and electromagnetic fields are excited and higher-order modes $(n \geq 2)$ are suppressed. The index $m$ was fixed at zero because it did not affect the stored energy and the $\mathrm{Q}$ factor.

The $\mathrm{Q}$ factor of the lossless and self-resonant antenna $Q_{\text {sr }}$ is defined as [13]

$$
Q_{\mathrm{sr}}=\omega \frac{W_{e}+W_{m}}{P_{\mathrm{r}}},
$$

where $\omega$ is the angular frequency, $P_{\mathrm{r}}$ is the total radiated power, and $W_{e}$ and $W_{m}$ denote the stored electric and magnetic energy, respectively. The radiated power and stored energy can be separated into the TM mode part and the TE mode part as follows:

$$
\begin{aligned}
P_{\mathrm{r}} & =P_{\mathrm{r}}^{\mathrm{TM}}+P_{\mathrm{r}}^{\mathrm{TE}} \\
W_{e} & =W_{e}^{\mathrm{TM}}+W_{e}^{\mathrm{TE}} \\
W_{m} & =W_{m}^{\mathrm{TM}}+W_{m}^{\mathrm{TE}} .
\end{aligned}
$$

Substituting Eqs. (2)-(4) into Eq. (1), the Q factor can be rewritten as

$$
Q_{\mathrm{sr}}=\left(Q_{e}^{\mathrm{TM}}+Q_{m}^{\mathrm{TM}}\right) \times \frac{P_{\mathrm{r}}^{\mathrm{TM}}}{P_{\mathrm{r}}^{\mathrm{TM}}+P_{\mathrm{r}}^{\mathrm{TE}}}+\left(Q_{e}^{\mathrm{TE}}+Q_{m}^{\mathrm{TE}}\right) \times \frac{P_{\mathrm{r}}^{\mathrm{TE}}}{P_{\mathrm{r}}^{\mathrm{TM}}+P_{\mathrm{r}}^{\mathrm{TE}}}
$$

where

$$
Q_{e}^{\mathrm{TM}}=\omega \frac{W_{e}^{\mathrm{TM}}}{P_{\mathrm{r}}^{\mathrm{TM}}}, \quad Q_{m}^{\mathrm{TM}}=\omega \frac{W_{m}^{\mathrm{TM}}}{P_{\mathrm{r}}^{\mathrm{TM}}}, \quad Q_{e}^{\mathrm{TE}}=\omega \frac{W_{e}^{\mathrm{TE}}}{P_{\mathrm{r}}^{\mathrm{TE}}}, \quad Q_{m}^{\mathrm{TE}}=\omega \frac{W_{m}^{\mathrm{TE}}}{P_{\mathrm{r}}^{\mathrm{TE}}} .
$$

The $\mathrm{Q}$ factor is the sum of contributions from both the TM and TE modes. Each $Q_{e, m}^{\mathrm{TM}, \mathrm{TE}}$ is calculated as mentioned in Ref. [4] in terms of the spherical Bessel functions. The self-resonant $\mathrm{Q}$ factor $Q_{\mathrm{sr}}$ has been described in Ref. [11] by Eq. (5); however, $Q_{\text {sr }}$ in the elementary function was not described in this work. The ratio of the TE-to-TM radiated power of the resonance antenna $[8,14]$ for $Q_{\mathrm{sr}}$ is given by 


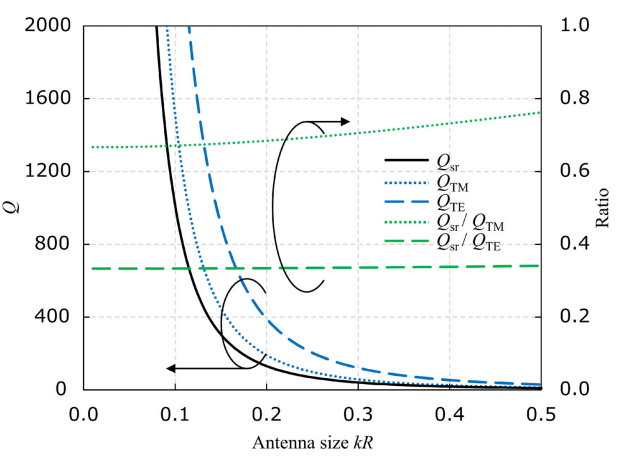

(a) Single-mode and self-resonant Q factor.

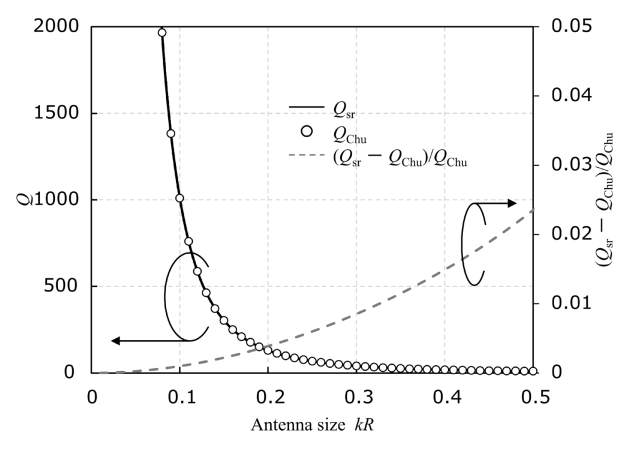

(b) Self-resonant and Chu Q factor.

Fig. 1. Q factor of lossless self-resonant spherical surface antenna $Q_{\mathrm{sr}}$.

$$
\frac{P_{\mathrm{r}}^{\mathrm{TM}}}{P_{\mathrm{r}}^{\mathrm{TE}}}=\frac{-\mathrm{j}_{1}(k R) \mathrm{y}_{1}(k R)+k R \mathrm{y}_{0}(k R) \mathrm{j}_{1}(k R)}{\mathrm{j}_{1}(k R) \mathrm{y}_{1}(k R)-k R \mathrm{y}_{1}(k R) \mathrm{j}_{0}(k R)}
$$

where $\mathrm{j}_{n}$ and $\mathrm{y}_{n}$ are the spherical Bessel functions of the first kind and second kind of the $n$-th order, and $k$ denotes the free space wavenumber. On substituting Eq. (7) into (5) and using Rayleigh's formulas [12], $Q_{\text {sr }}$ becomes the explicit form of elementary functions; this can be rewritten as

$$
\begin{aligned}
Q_{s r}= & \frac{1}{2}\left[\left\{4(k R)^{4}-12(k R)^{2}+2\right\} \sin ^{4}(k R)+\left\{10(k R)^{3}-8(k R)\right\} \cos (k R) \sin ^{3}(k R)\right. \\
& +\left\{4(k R)^{6}-6(k R)^{4}+12(k R)^{2}-2\right\} \sin ^{2}(k R) \\
& +\left\{-2(k R)^{7}+4(k R)^{5}-5(k R)^{3}+4(k R)\right\} \cos (k R) \sin (k R) \\
& \left.-2(k R)^{6}+(k R)^{4}-2(k R)^{2}\right] \\
& \cdot\left[\left\{2(k R)^{5}-(k R)^{3}\right\} \sin ^{2}(k R)+\left\{2(k R)^{4}-(k R)^{6}\right\} \cos (k R) \sin (k R)-(k R)^{5}\right]^{-1} .
\end{aligned}
$$

The self-resonant $\mathrm{Q}$ factor $Q_{\mathrm{sr}}$ in the exact form in Eq. (8) can be approximated by

$$
Q_{\mathrm{sr}} \approx \frac{1}{(k R)^{3}}+\frac{11}{10} \frac{1}{k R}
$$

where $k R \ll 1$. This expression coincides with the formula using the equivalent circuit method [9].

Fig. 1(a) shows the single and self-resonant Q factor of the lossless spherical antenna. This figure indicates that the $Q_{\mathrm{sr}}$ is approximately a third and two-thirds of the TE and TM single-mode $\mathrm{Q}$ factors $\left(Q_{\mathrm{TE}}\right.$ and $\left.Q_{\mathrm{TM}}\right)$, respectively. The approximated formula in Eq. (9) is shown in Fig. 1(b) and is close to the Chu limit $Q_{\text {Chu }}$ for the small antenna region $(k R \leq 0.5)$. $Q_{\text {sr }}$ includes the stored energy inside the antenna, whereas the Chu limit does not consider the internal energy.

\section{Effective $Q$ factor for spherical surface antenna}

The effective $\mathrm{Q}$ factor for self-resonant spherical surface antennas $Q_{\text {eff }}$ is defined as $Q_{\text {eff }}=\eta Q_{\text {sr }}$, where $\eta$ denotes the radiation efficiency of the spherical surface antenna. $\eta$ can be rewritten as $\eta=\left(1+P_{l} / P_{r}\right)^{-1}$ where $P_{l} / P_{r}$ is the ratio of the dissipated power to the radiated power. $P_{l} / P_{r}$ is calculated under the assumption of uniform current distribution in the skin depth $D[8]$ as 


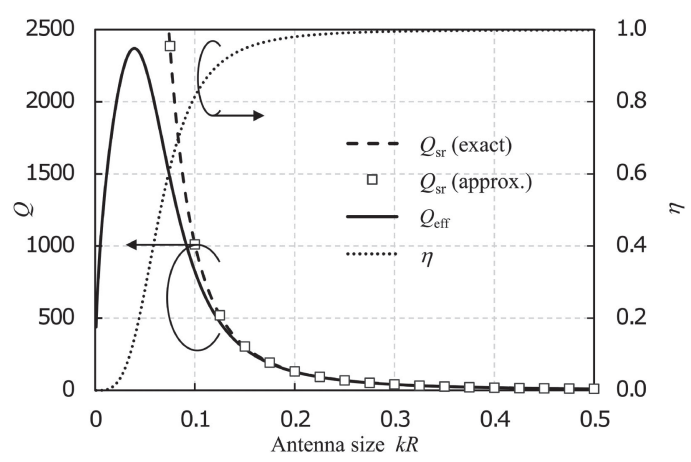

Fig. 2. Effective $\mathrm{Q}$ factor $Q_{\text {eff }}$ and radiation efficiency $\eta$.

$$
\begin{aligned}
\frac{P_{l}}{P_{r}}= & \frac{k}{Z_{0} \sigma} \frac{1}{(k R)(k R-k D)(k D)+\frac{(k D)^{3}}{3}} \frac{P_{\mathrm{r}}^{\mathrm{TM}}}{P_{\mathrm{r}}^{\mathrm{TE}}+P_{\mathrm{r}}^{\mathrm{TM}}} \\
& \times\left[\left\{\mathrm{j}_{0}(k R)-\frac{\mathrm{j}_{1}(k R)}{k R}\right\}^{-2}+\frac{P_{\mathrm{r}}^{\mathrm{TE}}}{P_{\mathrm{r}}^{\mathrm{TM}}}\left\{\mathrm{j}_{1}(k R)\right\}^{-2}\right]
\end{aligned}
$$

where $Z_{0}$ is the free-space impedance. $Q_{\text {eff }}$ is plotted in Fig. 2 with the $\eta$ of Eq. (10), the $Q_{\text {sr }}$ of Eq. (8), and the approximated $Q_{\text {sr }}$ of Eq. (9) against the antenna size. For this calculation, antennas were assumed to be made of copper $(\sigma=$ $5.8 \times 10^{7} \mathrm{~S} / \mathrm{m}$ ) and have a radius of $R=0.04 \mathrm{~m}$. In the region of $k R>0.05$, the $Q_{\text {eff }}$ is similar to the $Q_{\text {sr }}$ owing to the high radiation efficiency, whereas a significant difference is observed in the region of $k R<0.05$.

Eq. (10) can be expanded in the Laurent series and approximated by

$$
\frac{P_{l}}{P_{r}} \approx \sqrt{\frac{\omega \varepsilon_{0}}{2 \sigma}}\left(\frac{3}{(k R)^{4}}+\frac{3}{10} \frac{1}{(k R)^{2}}\right)
$$

where $\varepsilon_{0}$ denotes the free-space permittivity. With the aid of Eqs. (9) and (11), the approximated effective $\mathrm{Q}$ factor can be expressed as

$$
Q_{\text {eff }} \approx \frac{10 k R+11(k R)^{3}}{10(k R)^{4}+\sqrt{\frac{\omega \varepsilon_{0}}{2 \sigma}}\left(30+3(k R)^{2}\right)} .
$$

This newly derived formula has an error of less than $2 \%$ for the exact value in the region of $k R<0.5$ because the approximated radiation efficiency and effective $\mathrm{Q}$ factor is accurate in the same region.

\section{Numerical validation}

Fig. 3(a) shows the numerical validation of $Q_{\text {eff }}$. The effective $\mathrm{Q}$ factors of the infinitesimal dipole and loop antenna [7] are indicated by $Q_{\mathrm{lbe}}$ and $Q_{\mathrm{lbm}}$, respectively. $Q_{\mathrm{lbe}}$ is close to $Q_{\mathrm{eff}}$, whereas $Q_{\mathrm{lbm}}$ is smaller than $Q_{\mathrm{eff}}$. This is due to the relatively large radiation efficiency of the infinitesimal dipole antenna and the extremely small efficiency of the infinitesimal loop antenna. $Q_{\text {Carl }}$ in Fig. 3(a) is the effective $Q$ factor calculated by the equivalent circuit method [9]. Two current sheets radiating inside and outside of the sphere are assumed for $Q_{\text {Carl }}$, whereas $Q_{\text {eff }}$ is calculated with one current sheet. This area of the current sheet causes a 


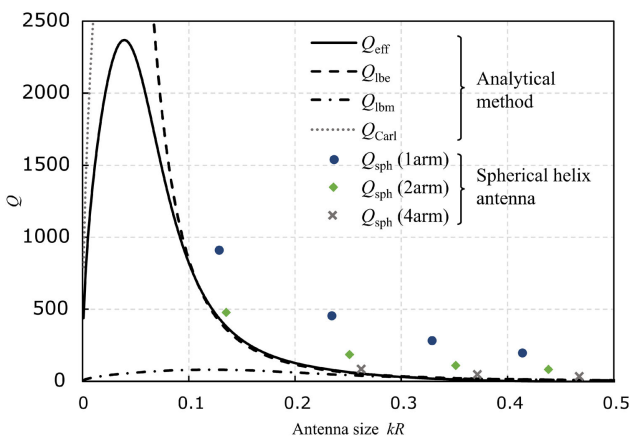

(a) Comparison of effective $\mathrm{Q}$ factors calculated via various methods.

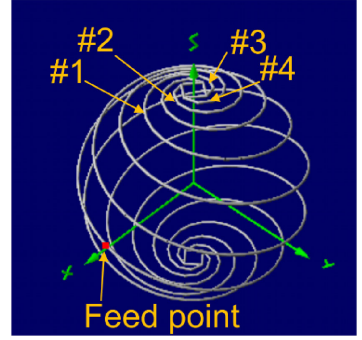

(b) Example of spherical helix antenna. (four arms)

Fig. 3. Validation of effective $Q$ factor using the analytical and numerical method.

difference in the radiation efficiency and the effective Q factor. Both $Q_{\text {eff }}$ and $Q_{\text {Carl }}$ have the order of $(k R)^{1}$.

The effective $\mathrm{Q}$ factor of one-, two-, and four-arm spherical helix antennas $Q_{\text {sph }}$ shown in Fig. 3(b) were also calculated numerically and are plotted in Fig. 3(a). The method of moment (NEC2 engine) was used for this numerical simulation. As the current distribution of these antennas was similar to the ideal spherical current sheet, the result approached $Q_{\text {eff }}$ as the number of wires increased.

It is obvious that the formula can estimate the effective $Q$ factor of a spherical helix antenna. As the spherical expansion limits the shape of the antenna to a sphere, the formula is limited to spherical-type antennas. The resultant formula, however, may provide a rough estimate for general small antennas because a spherical surface antenna is considered the simplest and most well-analyzed model of small antennas.

\section{Conclusion}

In this paper, we described the exact self-resonant $\mathrm{Q}$ factor $Q_{\mathrm{sr}}$ and approximated an effective $\mathrm{Q}$ factor $Q_{\text {eff }}$ using the stored energy calculated by spherical wave expansion. The approximated expression of $Q_{\text {eff }}$ was derived for the first time. It was confirmed that $Q_{\mathrm{sr}}$ is smaller than the single-mode $\mathrm{Q}$ factor and is close to the Chu limit. The simulated results of the spherical helix antennas demonstrated that the effective $\mathrm{Q}$ factor of these antennas approaches $Q_{\text {eff }}$. Moreover, comparisons between $Q_{\text {eff }}$ and the previous results obtained via the equivalent circuit method validate the value obtained for $Q_{\text {eff }}$. In a future study, the radiation efficiency and the effective $\mathrm{Q}$ factor will be measured and compared with these results.

\section{Acknowledgments}

A portion of this study has been supported by JSPS KAKENHI Grant Number JP18K13760. 